\title{
Thoughts and Progress
}

\section{Fabrication of Functional Cardiac, Skeletal, and Smooth Muscle Pumps In Vitro}

\author{
Rebecca Evers, Luda Khait, and Ravi K. Birla \\ Artificial Heart Laboratory, Division of Cardiac \\ Surgery, University of Michigan, Ann Arbor, \\ MI, USA
}

\begin{abstract}
Cardiovascular disease is one of the leading causes of death in the United States, and new treatments need to be developed in order to provide novel therapies. Tissue engineering aims to develop biologic substitutes that restore tissue function. The purpose of the current study was to construct cell-based pumps, which can be viewed as biologic left ventricular assist devices. The pumps were fabricated by culturing cardiac, skeletal, and smooth muscle cells within a fibrin gel and then each 3-D tissue construct was wrapped around a decellularized rodent aorta. We described the methodology for pump fabrication along with functional performance metric, determined by the intra-luminal pressure. In addition, histologic evaluation showed a concentric organization of components, with the muscle cells positioned on the outermost surface, followed by the fibrin gel and the decellularized aorta formed the innermost layer. Though early in development, cell-based muscle pumps have tremendous potential to be used for basic and applied research, and with further development, can be used clinically as cell-based left ventricular assist devices. Key Words: Tissue engineering-Regenerative medicine-Heart-Cell culture-Pressure-Decellular scaffold-Tissue construct-3-D.
\end{abstract}

Heart failure can be a result of many physiologic abnormalities, including myocyte death and dysfunction, ventricular remodeling postmyocardial infarction or any of these combinations (1). Current therapies to treat heart failure are limited to surgical transplantation, pharmaceutical intervention, and mechanic cardiac support (2). Even though these treatments have improved the quality of patient care, there are numerous limitations to these approaches.

doi:10.1111/j.1525-1594.2010.01007.x

Received August 2009; revised December 2009.

Address correspondence and reprint requests to Dr. Ravi K. Birla, Section of Cardiac Surgery, University of Michigan, MSRB I, A510E, 1150 West Medical Center Drive, Ann Arbor, MI 48109, USA.E-mail: rbirla@umich.edu
For example, mechanic circulatory support devices such as left ventricular assist devices (LVADs) are used as a bridge to cardiac transplantation; however, these devices suffer due to thrombogenicity, rejection, and infection (3). The ability to engineer a cellbased cardiac pump may provide an alternative to mechanic LVADs. A cell-based cardiac pump would be composed of a hollow chamber surrounded by contracting cardiac cells with one-way valves for unidirectional flow (4). The cell-based alternatives to mechanic LVADS would offer a higher degree of immune tolerance and would reduce the need for long-term anticoagulant and immunosuppression therapy. The goal of this study is to fabricate functional 3-D pumps from smooth, skeletal, and cardiac muscle cells, and to evaluate each pump's contractile capabilities in response to chemical and mechanic stimulation.

\section{MATERIALS AND METHODS}

Approval for animal use was granted by the University Committee for the Use and Care of Animals in accordance with the "Guide for the Care and Use of Laboratory Animals" (NIH publication 86-23, 1996). Aortas were excised from adult SpragueDawley rats and were completely decellularized using a detergent-based process, according to a published protocol (5). Primary cardiac myocytes were isolated from 2- to 3-day old Sprague-Dawley rat hearts using an established protocol (6). Skeletal muscle cells were isolated from rat soleus muscle using a previously published protocol (7). Vascular smooth muscle cells were purchased from Cambrex (Walkersville, MD, USA; catalog no. CC-2571, lot no. 4F1292) and cultured based on a previously published protocol (8). In order to fabricate 3-D pumps, tissue culture plates were prepared on the surface of a fibrin gel (Fig. 1A,B). Cardiac cells were plated at $1 \times 10^{6}$ cells/plate and skeletal/smooth muscle cells were plated at $0.5 \times 10^{6}$ cells/plate. The muscle cells were maintained under sterile cell culture conditions for a period of 4-5 days with media changes every other day. After the cell monolayer had compacted the fibrin gel to approximately $50 \%$ of the original volume, the decellularized aortas were placed at the 

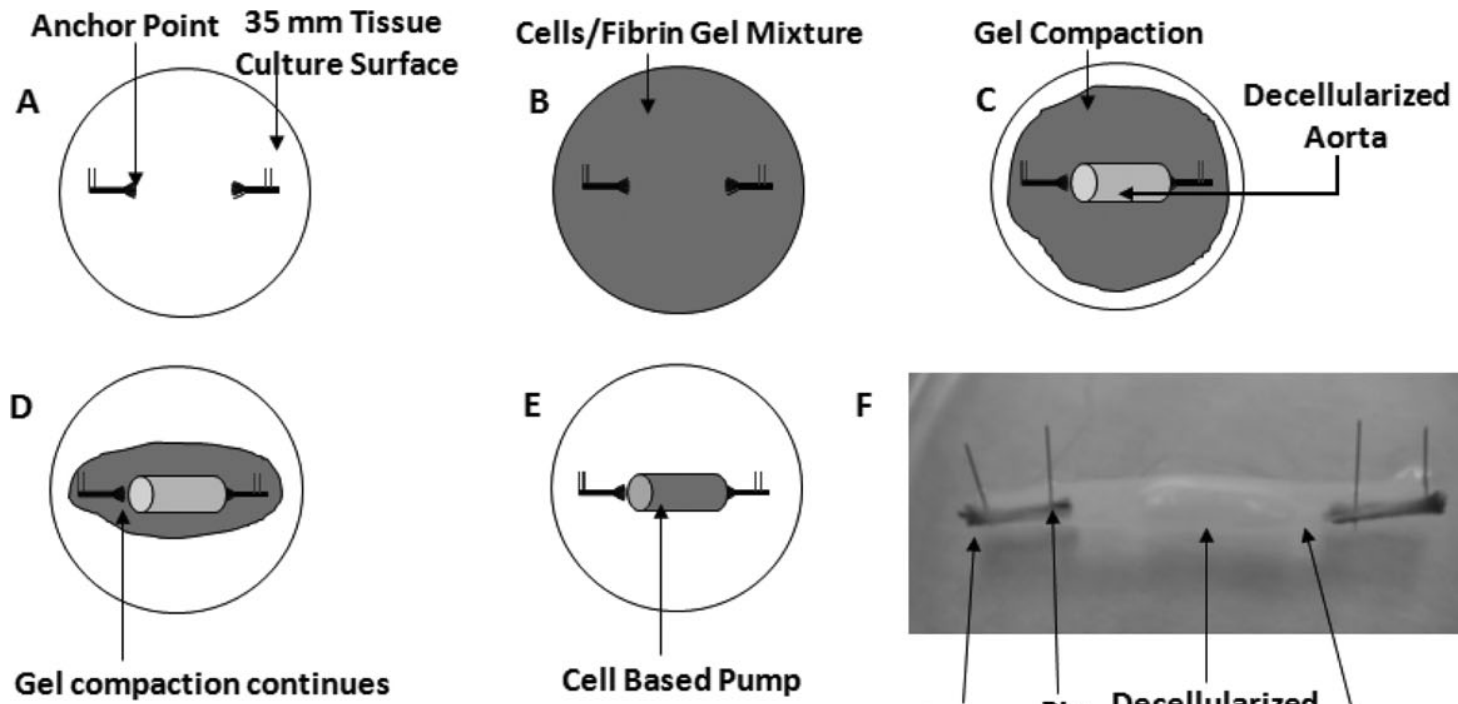

$\mathbf{F}$

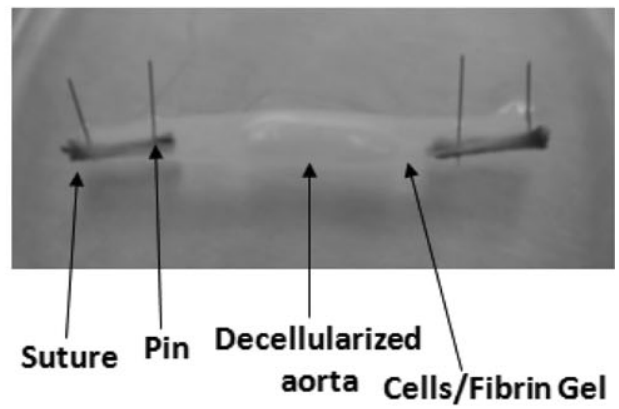

G

H
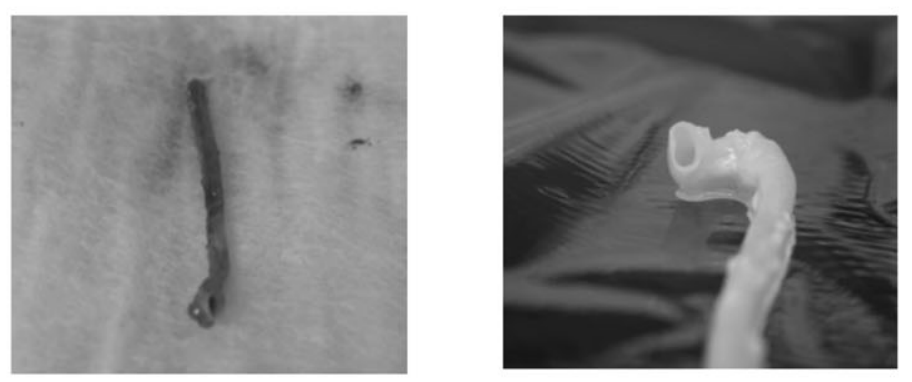

I

J
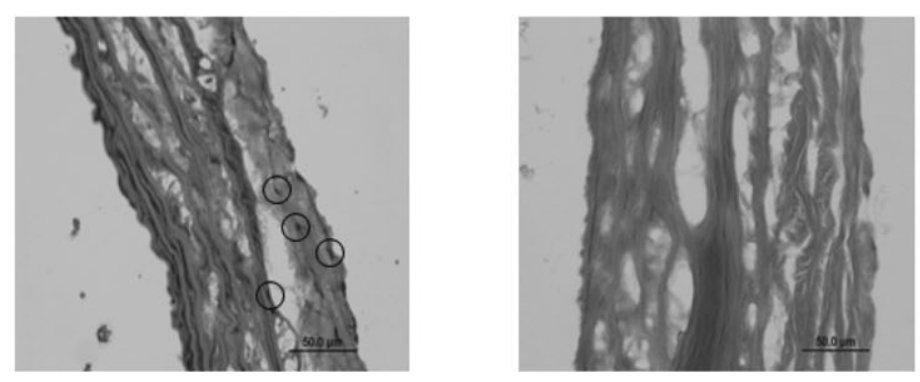

FIG. 1. Methodology for pump formation. (A) Tissue culture surfaces were coated with polydimethylsiloxane and anchor points (size 0 braided silk sutures), pinned to the center of plate using 0.1-mm diameter mintuetin pins. (B) Primary cells (cardiac, skeletal, or smooth) were suspended in a fibrin gel and plated on the surface of the tissue culture plate. (C) Once gel compaction started (typically 3-4 days after initial cell plating), a decellularized aorta was pinned to the center of the plate. (D) Gel compaction continued, with the decellularized aorta positioned at the center of culture surface. (E) Attachment of the cell monolayer to the decellularized aorta resulted in the formation of a cell-based pump. (F) Photograph of a cell-based cardiac pump formed utilizing primary cardiac myocytes. (G-J) Decellularization of rat aortas-detergent-based process to remove cellular components was used. (G) Freshly isolated aorta. (H) Decellularized aorta. (I) Hematoxylin and eosin (H\&E) stain of freshly isolated aorta-cell nucleus identified by circle. (J) H\&E stain of de-cellularized aortas-no cells can be seen.

center of the plates (Fig. 1C). The muscle cells continued to compact the fibrin gel toward the center of the culture surface (Fig. 1D). At the center of the plate, the cell monolayer was physically wrapped around the decellularized aortas using sterile forceps (Fig. 1E). The constructs were maintained for an additional 7 days to promote pump formation (Fig. $1 \mathrm{~F})$. Miniature pressure transducers were used to 
measure intra-luminal pressure. For cardiac pumps, intra-luminal pressure was recorded in response to spontaneous contractions, in the absence of any electric or chemical stimulation. For skeletal muscle pumps, intra-luminal pressure was recorded in response to electric stimulation, using an impulse of pulse width of $1.2 \mathrm{~ms}$, voltage of $20 \mathrm{~V}$, and frequencies of 1 and $60 \mathrm{~Hz}$. For smooth muscle pumps, the intraluminal pressure was recorded in response to chemical stimulation using $10 \mu \mathrm{M}$ of norepinephrine (NE). All pressure measurements were conducted at a single time point of 14 days after initial cell plating. Following mechanical testing, the cell-based pumps were fixed and stained using hematoxylin and eosin (H\&E).

\section{RESULTS}

\section{Decellularization procedure}

We used a detergent-based decellularization protocol developed by our group. Using this protocol, we were able to completely remove cellular and nucleic material, while keeping the extracellular matrix intact. Macroscopic changes in the rodent aorta were observable as a loss of cellularity, which led to changes in tissue appearance (Fig. 1G,H). The efficiency of the decellularization protocol was assessed using H\&E stain, and the absence of nucleic material was observed (Fig. 1I,J); an intact extracellular matrix can also be seen. This decellularization procedure was reproducibly repeated for $25+$ rodent aortas.

\section{3-D culture of cardiac, skeletal, and smooth muscle cells}

Previously described methods were used to culture primary cardiac, skeletal, and smooth muscle cells within a 3-D fibrin gel. Culture of primary cardiac cells within a fibrin gel resulted in a spontaneously contracting tissue construct, with macroscopic contractions observable under an inverted microscope at low magnification $(10 \times)$. Culture of primary skeletal muscle cells within a fibrin gel resulted in cell proliferation, seen using an inverted microscope, which demonstrated an increase in the surface area populated by skeletal myocytes. Changing the culture environment by reducing the media serum concentration promoted the formation of contracting myotubes; this led to fibrin gel compaction. Culture of primary smooth muscle cells (derived from an aortic source) resulted in cell proliferation and gel compaction; there were no macroscopic signs of spontaneous contractions and/or cell fusion. For each of the three cell types (cardiac, skeletal, and smooth), we fabricated $10+$ plates by culturing within a 3-D fibrin gel and the behavior of the cells was consistent.

\section{3-D pump formation}

In order to fabricate 3-D pumps, $8 \mathrm{~mm}$ segments of decellularized rat aortas were placed in the center of the compacting fibrin gel, with cardiac, skeletal, and/or smooth muscle cells (as described in Materials and Methods). Gel compaction resulted in radial movement of the fibrin gel toward the vessel segment. The fibrin gel was then physically wrapped around the decellularized vessel and subsequent culture resulted in 3-D pump formation. Over time, in culture, the cell monolayer became more compact and more closely associated with the aorta (evident under an inverted microscope). Spontaneous contractions (frequency of $1-2 \mathrm{~Hz}$ ) of the 3-D pumps were observed only for the constructs fabricated using cardiac cells; these contractions were visible under an inverted microscope. A total of $30+$ pumps were fabricated using this procedure with an $80 \%$ success rate, with success being defined as the formation of 3-D construct. In 10\% of the pumps, there was contamination which likely resulted from the physical wrapping of the fibrin gel around decellularized vascular segments. In another $10 \%$ of the cases, the fibrin gel did not adhere to the vascular segments; rather, the 3-D fibrin gel with the muscle cells remained in culture as an independent entity.

\section{Functional evaluation of 3-D pumps-intra-luminal pressure}

The intra-luminal pressure of 3-D cardiac pumps was measured during spontaneous contractions and was on average $0.12 \mathrm{~mm} \mathrm{Hg}$ with the average frequency of $1.5 \mathrm{~Hz}$ (Fig. 2A). The smooth muscle pumps were only excitable upon chemical stimulation and in response to $10 \mu \mathrm{M} \mathrm{NE}$, an average pressure of $0.008 \mathrm{~mm} \mathrm{Hg}$ was obtained (Fig. 2B). For the skeletal pump, there were no spontaneous contractions and intra-luminal pressure was only recorded upon electric stimulation (Fig. 2C,D). When the 3-D skeletal pumps were electrically stimulated at a frequency of $1 \mathrm{~Hz}$, the number of pressure waves correlated with the pacing frequency; individual twitch contractions were observed (Fig. 2C). However, when the pacing frequency was increased to $60 \mathrm{~Hz}$, tetanus contractions were observed, with a magnitude of $0.025 \mathrm{~mm} \mathrm{Hg}$ (Fig. 2D).

\section{Histologic evaluation of 3-D pumps}

Histologic evaluation of the pumps showed the presence of an intact acellular vessel, with concentric 
A

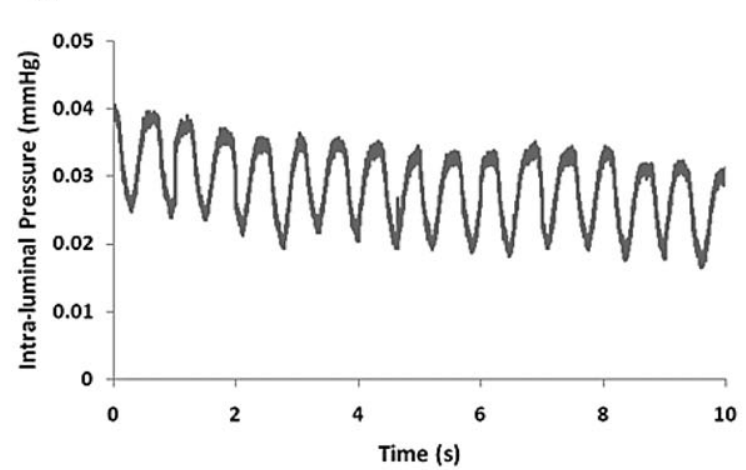

C

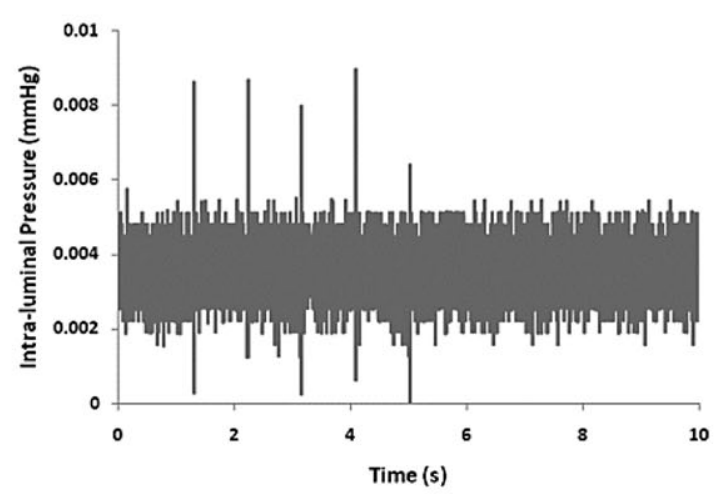

E

\section{B}

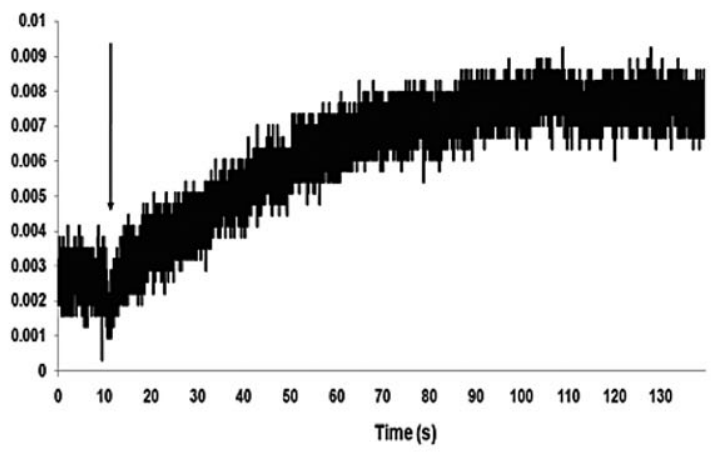

D

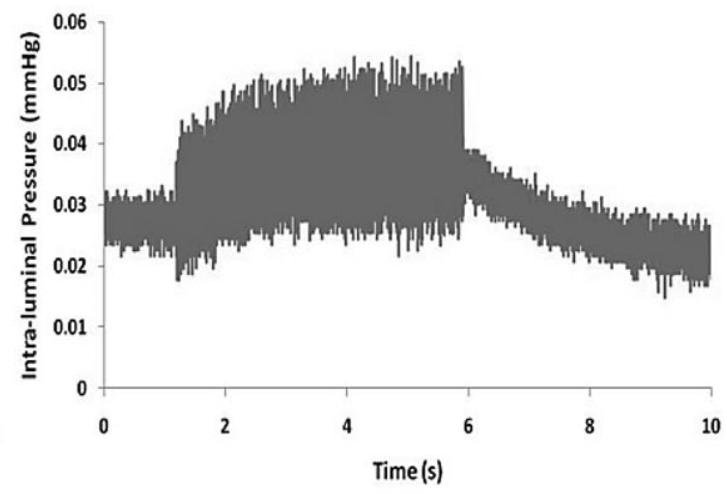

$\mathbf{F}$

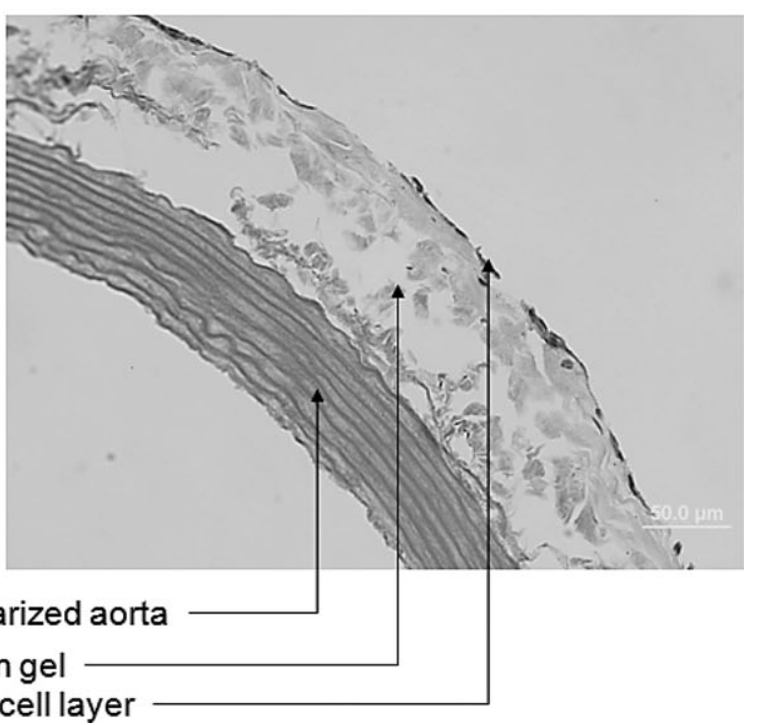

FIG. 2. Functional performance of cell-based pumps. (A) Cardiac pump-in the absence of electric stimulation, the cardiac pumps were capable of generating spontaneous contractions. The average pressure was on the order of $0.014 \mathrm{~mm} \mathrm{Hg}$ and the frequency of spontaneous contractions was approximately $1.5 \mathrm{~Hz}$. (B) Smooth muscle pump-the smooth muscle pumps were stimulated with $10 \mu \mathrm{M}$ of norepinephrine (NE) and the intra-luminal pressure recorded for a period of $140 \mathrm{~s}$. The point at which the NE was added is indicated by an arrow. The average pressure was recorded to be $0.008 \mathrm{~mm} \mathrm{Hg}$. (C-D) Skeletal muscle pump-the skeletal muscle pumps were subjected to electric stimulation, using a pulse width of $1.2 \mathrm{~ms}$, voltage of $20 \mathrm{~V}$, and two different frequencies: (C) $1 \mathrm{~Hz}$ and (D) $60 \mathrm{~Hz}$. The average pressure was $0.025 \mathrm{~mm} \mathrm{Hg}$ at $60 \mathrm{~Hz}$. (E) Histologic evaluation of muscle-based pump-a segment of decellularized aortic tissue was used as the scaffold material, with primary muscle cells culture in a fibrin gel, wrapped around the peripheral surface. (F) Tri-layer structure of 3-D pump-a high magnification image of the muscle-based pump showing the organization of the decellular aorta, fibrin gel, and muscle cells. Images are for skeletal muscle pump and are representative of pumps fabricated with all three muscle types (cardiac, skeletal, and smooth). 
alignment of a fibrin gel followed by muscle cells on the outer surface of the 3-D pump (Fig. 2E). The acellular vessel was completely intact and retained its 3-D architecture, and it was evident that no cellular material was present within the vascular segment (Fig. 2F). The cell component of the pump was separated from the vascular segment by the fibrin gel; this prevented physical contact between the cells and the vascular segment (Fig. 2F). However, cells were evident around the entire circumferential surface of the 3-D pumps. This arrangement of the vascular segment, fibrin gel, and muscle cells was independent of the cell type being used and was a function of the methodology implemented for the formation of 3-D pumps.

\section{DISCUSSION AND CONCLUSIONS}

In this study, we describe a novel method to bioengineer 3-D cell-based pumps using cardiac, skeletal, and smooth muscle cells. We describe the methodology for pump formation and demonstrate functional performance based on intra-luminal pressure. In this section, we discuss the following five topics: (i) the need for cell-based pumps; (ii) methodology for pump formation; (iii) functional performance of cell-based pumps; (iv) technological advancements required to develop these pumps further; and (v) path to clinical applications.

\section{Need for cell-based pumps}

In this study, we describe methodology to fabricate functional 3-D cell-based pumps. The concept of cellbased pumps has not been extensively explored in the research literature and very few scientific publications can be found discussing this topic. Therefore, prior to presenting our discussion for this study, it would be useful to describe what exactly cell-based pumps are and how can they be used in a research and/or clinical setting. Cell-based pumps can be viewed as biologic devices capable of generating pressure upon spontaneous or electrically/chemically induced contractions. Although these devices are early in development, they can be conceptually viewed as biologic alternatives to LVADs. LVADs are used as a bridge to heart transplantation and function by pumping blood directly from the apex of the heart to the aorta, completely bypassing the failing left ventricle. However, LVADs are devices and suffer from thrombogenicity problems, something cellbased alternatives would reduce.

\section{Methodology for 3-D pump formation}

In order to fabricate 3-D pumps, primary muscle cells were cultured within a 3-D fibrin gel and then physically wrapped around a decellularized aortic vascular segment. This resulted in the fibrin gel being placed in close proximity to the decellular scaffold, leading to attachment of the fibrin gel to the scaffold and the formation of 3-D cell-based pumps. This method is simple, easy to implement and reproducible, and most importantly, leads to the formation of functional pumps. However, one limitation is the distance between the muscle cells and acellular matrix, with fibrin gel occupying a substantial volume in between these two layers. This distance between the muscle cells and acellular scaffolds dampens the contractile activity of the cells, thereby reducing scaffold displacement; this reduces pump functionality, measured by intra-luminal pressure. This is the most likely explanation of low pressures obtained in this study. While adequate for a proof of concept study, subsequent work will need to focus on functional integration between the muscle cells and the scaffold. This can be accomplished by promoting fibrin degradation or replacing the 3-D culture system from a fibrin-based model to one based on self organization strategies.

\section{Functional performance of cell-based pumps}

The functional performance of the 3-D pumps, fabricated using any of the three muscle cell types, was considerably lower than that of mammalian tissue. This could partly be due to the concentric organization of the acellular scaffold, fibrin gel, and muscle cells, with the fibrin gel presenting a barrier between the cells and the scaffold. Another possible explanation for the low functional performance of the pumps is the single layer of cells which was used in this study. An increase in intra-luminal pressure of the 3-D pumps will require multi-layering of the muscle cells coupled with functional integration of the cells with the scaffold, without physical hindrance of the intervening fibrin gel. While these strategies would be required for further development of the pump model, the current study does provide the foundation and serves as a proof of concept study.

\section{Technological advancements required to develop these pumps further}

In order to develop the pump model further, the functional performance of these devices will need to be closer to that of mammalian tissue. This can be accomplished by increasing the thickness of the muscle layer and promoting functional integration between the cells and the scaffold. While the exact number of cell layers required remains unknown, multiple layers will be required; vascularization may also be needed to support the metabolic activity of 
the increase in cell number. In addition, physiologic conditioning using mechanical stretch, pulsatile fluid flow, and chemical conditioning with growth factors will be required. It will also be important to control the electric activity of the muscle cells and externally control stimulation variables (frequency, introduction of rest periods for skeletal muscle) during longterm in vitro culture; bioreactors with embedded stimulators will be required.

\section{Path to clinical applications}

The muscle-based pumps are at a nascent stage of development, and considerable research needs to be invested prior to using these devices clinically. Perhaps the most important step in moving the pump model forward will be the use of stem cells in place of the primary cells used in this study. Several stem cell sources are currently under investigation and human embryonic stem cells, mesenchymal stem cells, and induced pluripotent cells are all potential sources for tissue engineering. From an applications standpoint, cardiac pumps can be used as actuators in robotic devices, where continuous performance is necessary. Skeletal and smooth muscle pumps will be suitable as miniature drug delivery devices, with skeletal pumps being suitable for infrequent delivery of high drug doses and smooth pumps being better for frequent delivery of low doses of specific molecule.

Acknowledgment: The authors would like to thank the Section of Cardiac Surgery at the University of Michigan for financial support.

\section{REFERENCES}

1. Cleutjens JP, Blankesteijn WM, Daemen MJ, Smits JF. The infarcted myocardium: simply dead tissue, or a lively target for therapeutic interventions. Cardiovasc Res 1999;44:232-41.

2. Hecker L, Birla R. Engineering the heart piece by piece: state of the art in cardiac tissue engineering. Regen Med 2007;2:125-44.

3. Stevenson LW, Kormos RL. Mechanical cardiac support 2000: current applications and future trial design. J Heart Lung Transplant 2001;20:1-38.

4. Khait L, Birla RK. Cell-based cardiac pumps and tissueengineered ventricles. Regen Med 2007;2:391-406.

5. Borschel GH, Kia KF, Kuzon WM Jr, Dennis RG. Mechanical properties of acellular peripheral nerve. J Surg Res 2003;114: 133-9.

6. Huang YC, Khait L, Birla RK. Contractile three-dimensional bioengineered heart muscle for myocardial regeneration. J Biomed Mater Res A 2007;80:719-31.

7. Dennis RG, Kosnik PE. Excitability and isometric contractile properties of mammalian skeletal muscle constructs engineered in vitro. In Vitro Cell Dev Biol Anim 2000;36:327-35.

8. Hecker L, Khait L, Welsh MJ, Birla RK. Bioengineering functional human aortic vascular smooth muscle strips in vitro. Biotechnol Appl Biochem 2008;50:155-63. 